\title{
Heart induction by Wnt antagonists depends on the homeodomain transcription factor Hex
}

\author{
Ann C. Foley and Mark Mercola ${ }^{1}$ \\ Stem Cell and Regeneration Program, Burnham Institute, La Jolla, California 92037, USA
}

\begin{abstract}
Inhibition of canonical Wnt/ß-catenin signaling by Dickkopf-1 (Dkk-1) or Crescent initiates cardiogenesis in vertebrate embryos. However, nearly nothing is known about the downstream effectors of these secreted Wnt antagonists or the mechanism by which they activate heart formation. Here we show that Wnt antagonists in Xenopus stimulate cardiogenesis non-cell-autonomously, up to several cells away from those in which

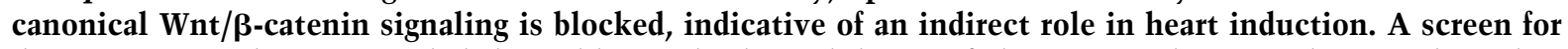
downstream mediators revealed that Dkk-1 and other inhibitors of the canonical Wnt pathway induce the homeodomain transcription factor Hex, which is normally expressed in endoderm underlying the presumptive cardiac mesoderm in amphibian, bird, and mammalian embryos. Loss of Hex function blocks both endogenous heart development and ectopic heart induction by Dkk-1. As with the canonical Wnt pathway antagonists, ectopic Hex induces expression of cardiac markers non-cell-autonomously. Thus, to initiate cardiogenesis, Wnt antagonists act on endoderm to up-regulate Hex, which, in turn, controls production of a diffusible heart-inducing factor. This novel function for Hex suggests an etiology for the cardiac malformations in Hex mutant mice and will make possible the isolation of factors that induce heart directly in the mesoderm.
\end{abstract}

[Keywords: Heart; Wnt; Hex; Dickkopf; Nkx2.5; Tbx5; Xenopus]

Received November 12, 2004; revised version accepted December 9, 2004.

The heart is among the earliest tissues specified in the mesoderm of vertebrate embryos and the genes and proteins responsible are of considerable interest because of their potential use for cardiomyocyte regeneration (for review, see Foley and Mercola 2004). Two secreted antagonists of Wnt signaling, Dickkopf-1 (Dkk-1) and Crescent, are potent inducers of ectopic cardiogenesis in nonheart mesoderm from either chick or Xenopus embryos (Marvin et al. 2001; Schneider and Mercola 2001; Tzahor and Lassar 2001). Other secreted Wnt antagonists, such as Frz-b and Szl, appear less active, probably owing to selectivity for the particular Wnts that must be prevented from signaling. The structurally distinct Dkk-1 and Crescent proteins both block signaling by preventing interaction of Wnts with receptors on the cell surface (for review, see Kawano and Kypta 2003). Intracellular inhibitors of the canonical Wnt/ $\beta$-catenin pathway initiate cardiogenesis (Schneider and Mercola 2001), but hearts are also induced in Xenopus mesodermal explants by Wnt-11 (Pandur et al. 2002a), which stimulates noncanonical signaling through Jun N-terminal kinase (JNK) and protein kinase-C (PK-C) and might also antagonize

${ }^{1}$ Corresponding author.

E-MAIL mmercola@burnham.org; FAX (858) 713-6274.

Article and publication are at http://www.genesdev.org/cgi/doi/10.1101/ gad.1279405. canonical signaling through $\beta$-catenin (Maye et al. 2004) in this setting. These studies indicate that inhibition of canonical Wnt/ $\beta$-catenin signaling and activation of noncanonical signaling are both important initiators of cardiogenesis in embryonic tissue in amphibians and amniotes, yet nearly nothing is known in any species about the genes and protein effectors that operate downstream of these pathways to initiate cardiogenesis. Their identification will be important not only for tissue engineering, but also to distinguish how heart induction differs from, and is coordinated with, other effects of Wnt signaling on cell fate and morphogenesis during embryogenesis.

In Xenopus embryos, Dkk1 and Crescent are produced within Spemann's Organizer, an important signaling center of the gastrula-stage embryo that eventually gives rise to the notochord and head mesoderm and expresses other signaling proteins involved in dorsoanterior patterning, including XNr-1, a homolog of the mouse Nodal protein, and BMP antagonists noggin and chordin (for review, see Harland and Gerhart 1997). The Organizer is clearly required for heart induction, as has been shown by extirpation studies (Sater and Jacobson 1990; Nascone and Mercola 1995); however, it cannot induce either native or ectopic heart tissue efficaciously unless accompanied by a small amount of underlying deep dorsoanterior endoderm (Nascone and Mercola 1995). Classical 
grafting studies also pointed out the heart-inducing properties of dorsoanterior endoderm in amphibians (Jacobson 1960; Jacobson and Duncan 1968; Fullilove 1970), and similar tissue extirpation and recombination experiments revealed heart-inducing activity in chick embryo anterior hypoblast (Yatskievych et al. 1997) and mouse embryonic anterior visceral endoderm (AVE) (Arai et al. 1997). The latter two are both extraembryonic but share expression of certain genes with amphibian dorsoanterior endoderm suggestive of common signaling properties (for discussion, see Bouwmeester et al. 1996).

Theoretically, Wnt antagonists might induce heart tissue in parallel with a signal from the dorsoanterior endoderm. One example of parallel signaling is a model (Marvin et al. 2001) based on chick embryo experiments (Sugi and Lough 1994; Schultheiss et al. 1997; Schlange et al. 2000; Marvin et al. 2001; Tzahor and Lassar 2001) in which the heart-forming region develops at the intersection where Wnt antagonists and BMP isoforms are presumed to act. Although BMPs are clearly necessary for Xenopus cardiogenesis, they are induced and needed only after the requirement for Wnt antagonists and endoderm has passed (Shi et al. 2000), suggesting that another inducing signal exists in the endoderm. An alternative model is that Wnt antagonists act on the endoderm to stimulate secretion of a molecule that diffuses into adjacent mesoderm to specify heart formation.
In this paper, we describe a genetic cascade that constitutes an indirect mode of action for Wnt antagonists in heart induction. We created mosaics of normally noncardiogenic ventroposterior mesendoderm consisting of cells that either express or do not express cell-autonomous inhibitors of canonical $\beta$-catenin signaling. Early heart markers were induced in cells that did not have inhibitors, and these were located up to several cell diameters removed from those with inhibitors. Wnt antagonists also induced the homeodomain transcription factor Hex (Newman et al. 1997; Thomas et al. 1998), but in contrast with heart genes, Hex transcripts were localized largely within the cells expressing the cell-autonomous inhibitors. Loss-of-function experiments showed that formation of both native hearts and hearts induced ectopically by Dkk-1 depends on the transcriptional-repressive function of Hex. The spatial and temporal patterns of Hex expression in tissues adjacent to the heartforming region in Xenopus, chick, and mouse embryos correlate with heart-inducing activities (Arai et al. 1997; Schneider and Mercola 1999; Yatskievych et al. 1999). We infer from these studies that Hex is likely to play an evolutionarily conserved role in heart induction, and we discuss the potential relationship to the complex heart malformations recently described in mice homozygous for a targeted mutant Hex allele (Hallaq et al. 2004).

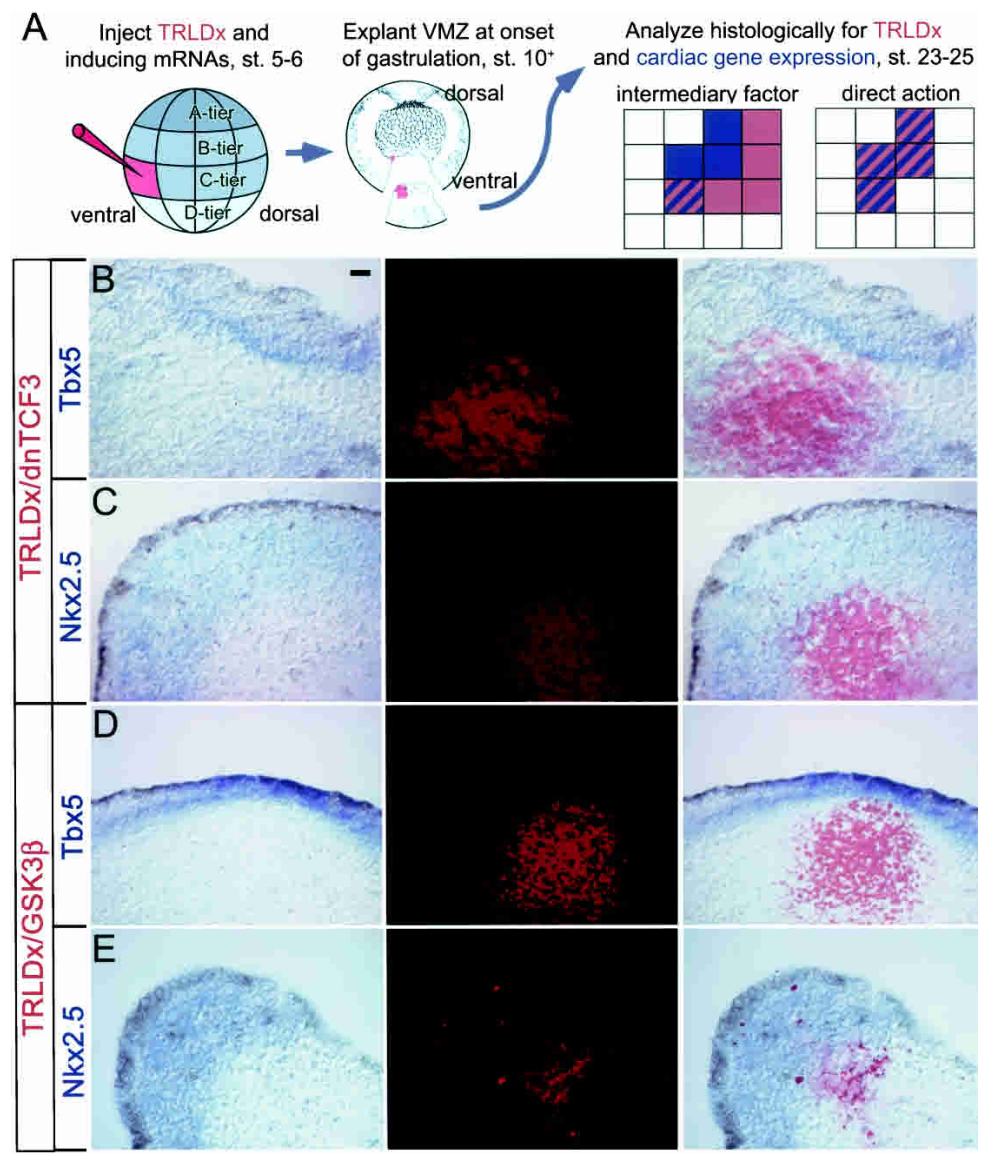

Figure 1. Non-cell-autonomous induction of Nkx2.5 and Tbx5 by Gsk3 $\beta$ and dnTCF3. (A) Embryos were injected into one ventral blastomere at the 16-32-cell stage with mRNAs encoding Gsk3 $\beta$ or dnTCF3 along with Texas Red lysinated dextran (TRLDx, 10,000 MW) as a lineage label. The normally noncardiogenic VMZ mesendoderm was explanted at the onset of gastrulation (stage 10.2510.5), cultured until cardiac tissue expressed Nkx2.5 and Tbx5 (stage 23-25), then stained for gene expression by in situ hybridization (blue). ( $B-$ $E)$ Examples of non-cell-autonomous gene induction on histological section. Injection of GSK3 $\beta$ or dnTCF3 mRNAs (with TRLDx) induced Tbx5 $(B, D)$ and $N k x 2.5(C, E)$. In situ hybridization is shown in the left panels, TRLDx fluorescence in the middle panels, and merged images on the right panels. Note that Tbx5- and Nkx2.5-positive cells were localized at a distance from lineage-labeled cells. Bar in $B$ represents $20 \mu \mathrm{M}$. 


\section{Results}

Induction of early cardiac markers by Gsk3 $\beta$ and dnTCF3 is non-cell-autonomous

To address whether Wnt antagonists act directly or indirectly to establish the cardiac mesoderm, we injected cell-autonomous inhibitors of the canonical Wnt/ $\beta$ cat signaling pathway, either Gsk3 $\beta$ (Larabell et al. 1997) or a dnTCF3 (Molenaar et al. 1996). mRNA encoding these genes was independently injected into one ventroposterior blastomere at the 16-32-cell stage, along with 10,000 MW Texas Red lysinated dextran (TRLDx), as a lineage marker in order to create a mosaic tissue having a minor fraction of cells expressing the cell-autonomous inhibitor of canonical Wnt/ $\beta$-catenin signaling (Fig. 1A). Explants of the ventroposterior marginal zone (VMZ) were dissected at stage 10.25-10.5 (Nieuwkoop and Faber 1967) and grown in culture until age-matched siblings reached stages 23-25. Explants were then processed by in situ hybridization for expression of either Nkx2.5 (Tonissen et al. 1994) or Tbx5 (Horb and Thomsen 1999), both of which mark the early heart field. For the most part, heart marker expression was observed several cell diameters away from the nearest TRLDx-expressing cell. Although this does not rule out the possibility that Wnt/ $\beta$-catenin antagonists are required in some cells that contribute to the heart field, this finding establishes that an intermediary factor, perhaps produced by endodermal cells, induces early cardiac gene expression. Cell nonautonomy was even more evident at later stages (approximately stage 35 ), after morphological movements had displaced cardiac cells a considerable distance from the majority of the lineage-labeled cells (Fig. 2), consistent with a heart-inducing requirement for the Organizer and deep dorsoanterior endoderm prior to the onset of tissue migration at gastrulation (Sater and Jacobson 1990; Nascone and Mercola 1995).

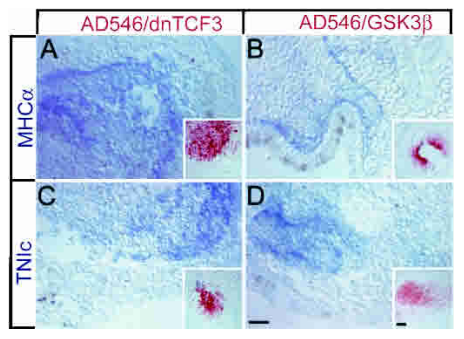

Figure 2. Non-cell-autonomous induction of late cardiac markers by Gsk3 $\beta$ and dnTCF3. $(A-D)$ Examples of induction of non-cell-autonomous induction of $M H C \alpha(A, B)$ and TnIc $(C, D)$ at stage 35 induced by dnTCF3 $(A, C)$ and Gsk3 $\beta(B, D)$. Lineage label (Alexa Fluor 546 Dextran [AD546])-positive cells were never found to overlap expression of the induced cardiac genes and were seen only rarely in adjoining cells; rather, they were displaced distantly in the explant during morphogenetic tissue movements that characterize gastrulation in intact embryos. Insets show that each explant contains robustly lineage-labeled cells in sections distant to those with cardiac gene expression. Bars in $D$ represent $20 \mu \mathrm{M}$.
Inhibition of Wnt/ $\beta$-catenin signaling induces Hex mRNA cell-autonomously

We had shown previously that the heart-inducing potency of the dorsoanterior endoderm maps spatially and temporally to the expression domains of the homeodomain-containing transcription factor Hex and the secreted factor Cerberus (Bouwmeester et al. 1996; Newman et al. 1997; Thomas et al. 1998; Schneider and Mercola 1999). This observation led us to test whether either of these candidate genes could induce expression of heart field markers by injecting capped, synthetic mRNA into one ventral blastomere of 4-8-cell stage Xenopus embryos and explanting and culturing VMZ explants until age-matched siblings reached stage 23-25 as above. Both Cerberus and Hex induced Nkx2.5 expression as visualized by in situ hybridization (9/9 for Cerberus; 40/68 for Hex). By quantitative, real-time RT-PCR, Cerberus increased $N k x 2.5$ expression in VMZ explants 12.6-fold $(P \leq 0.056, n=4)$ over that seen in control, $\beta$-gal-injected explants, and the level increased to 42.4-fold $(P \leq 0.096$, $n=4$ ) by coinjection of a plasmid expressing BMP7 from the cytoskeletal actin (CSKA) promoter, which directs protein expression after stage 11. Xenopus Cerberus is both a BMP and Wnt antagonist whose transient expression is extinguished at about stage $12-13$, before the cardiogenic requirement for BMP signaling (Bouwmeester et al. 1996; Shi et al. 2000); thus, CSKA-BMP7 was provided in an attempt to overcome the BMP inhibitory action of chronic Cerberus overexpression that might otherwise dampen heart induction. Hex achieved a 61.0fold $(P \leq 0.084, n=4)$ induction of $N k x 2.5$. In comparison, Dkk-1 injection increased expression on average 30.6-fold $(P \leq 0.003, n=11)$, indicating that the Hex response was particularly robust.

Next we injected either Gsk3 $\beta$ or dnTCF3 mRNAs into one ventral blastomere at the 4-8-cell stage to test if either Hex and/or Cerberus is regulated by canonical Wnt/ß-catenin inhibitors. Again, VMZs were explanted at stage $10.25-10.5$ and analyzed by quantitative RTPCR. Because these genes are expressed in dorsal to ventral gradients in the endoderm, special care was taken to ensure that only the ventral-most $60^{\circ}$ of the VMZ was analyzed. Quantitative RT-PCR revealed a significant increase in Hex expression in response to both dnTCF3 and Gsk3 $\beta$ (Fig. 3A), but no up-regulation of Cerberus (data not shown). Although this does not eliminate Cerberus as a potential inducer of cardiac tissue, it suggests that Hex rather than Cerberus is more likely to be an effector of Wnt antagonism.

In contrast to the induction of $N k \times 2.5$ and $T b \times 5$, the spatial domains of Hex expression induced by Gsk3 $\beta$ and dnTCF3 in the tissues largely overlapped the cells expressing the $\beta$-catenin signaling inhibitors in the mosaic assay (Fig. 3B-G'). As before, one ventral blastomere at the 16-32-cell stage was injected with mRNA encoding either Gsk3 $\beta$ or dnTCF3 with either $\beta$-gal or Alexa Fluor 546-dextran (AD546) as lineage label, and VMZ explants were isolated at stage 10.25-10.5 and processed for Hex in situ hybridization. Interestingly, some cells with the 

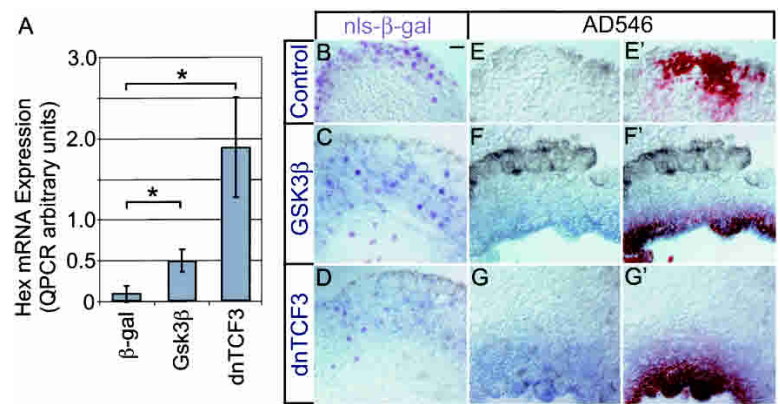

Figure 3. Induction of Hex mRNA by Gsk $3 \beta$ and $\operatorname{dnTCF} 3 .(A)$ Embryos were injected with synthetic mRNA for Gsk3 $\beta$ and dnTCF into one ventral blastomere of a 4-8-cell-stage embryo. Noncardiogenic VMZ explants were dissected at the onset of gastrulation (stage 10.25-10.5) then analyzed for mRNA levels by quantitative RT-PCR (mean of four experiments \pm standard error). The asterisk indicates a statistically significant difference ( $t$-test) from control $\beta$-gal-injected samples for VMZ explants expressing Gsk3 $\beta(P \leq 0.031)$ and dnTCF3 $(P \leq 0.032)$. $(B-D)$ Examples of induction visualized by in situ hybridization and staining for $\beta$-galactosidase. Control injection of nls- $\beta$-gal did not induce $\operatorname{Hex}(B)$, whereas Gsk3 $\beta(C)$ or dnTCF3 $(D)$ both induced Hex. In contrast to induction of cardiac markers, the spatial domain of Hex-positive cells largely overlapped the lineage-labeled cells. Note that some lineage-labeled cells in centers of explants do not express Hex, suggesting that induction is spatially constrained. $\left(E-G^{\prime}\right)$ Examples of induction visualized by in situ hybridization and AD546. Control injections of AD546 do not induce $\mathrm{Hex}(E)$, whereas injection of both GSK3b $\left(F, F^{\prime}\right)$ and dnTCF3 $\left(G, G^{\prime}\right)$ induce Hex. In situ hybridization staining is shown in $F$ and $G$, and merged in situ hybridization staining and AD546 fluorescence $\left(F^{\prime}, G^{\prime}\right)$ show nearly perfect overlap between cells expressing Hex and the lineage label. Bar in $B$ represents $20 \mu \mathrm{M}$.

inhibitors clearly did not show up-regulation of $\mathrm{Hex}$, often in the centers of the explants (e.g., Fig. 3C,D), suggesting that competence to respond to Wnt antagonist action is spatially constrained.

Induction of cardiac markers by Hex is non-cell-autonomous

If Hex were to act epistatically to Wnt/ $\beta$-catenin in a cascade that initiates heart formation, it should induce early cardiac markers at a distance from the cells in which it is expressed. To address this issue, Hex was injected along with a lineage label, either $\beta$-gal or TRLDx as before, and VMZ explants were grown in culture until age-matched siblings had reached stage 23-25, when they were analyzed for expression of $N k x 2.5$ and $T b x 5$ (Fig. 4). $N k \times 2.5$ or $T b x 5$ expression was clearly seen in the cells neighboring the lineage label, but there was no overlap. Figure 4 shows the results with TRLDx, and identical results were observed when nls- $\beta$-gal was used as the lineage label (data not shown). Thus, Hex induces a diffusible heart-inducing factor (or represses a suppressor).
Hex is necessary for heart formation epistatic to Dkk-1

To test for a functional role in normal heart induction, we injected a morpholino oligonucleotide (HexMo) as used previously to deplete Hex in the embryo (Smithers and Jones 2002). HexMo (20 ng) injected into two dorsoanterior blastomeres at the 4-cell stage consistently impeded heart formation, as assayed by reduction in incidence of $M H C \alpha$ and TnIc expression in dorsoanterior marginal zone (DMZ) explants (Fig. 5A,B, panels g,g') cultured until age-matched siblings had reached approximately stage 30 . DMZ explants, rather than intact embryos, were used because they robustly form hearts in culture and permit heart induction to be analyzed in response to inhibitors with less impact of potentially confounding effects of inhibitory signals from the ventroposterior region that might impinge on the heart field in foreshortened intact embryos; thus, DMZ explants constitute a more stringent test of an inhibitor that affects dorsoanterior patterning. In contrast to the effect with HexMo, both uninjected and control morpholino-injected (20 ng, ControlMo) DMZ explants developed hearts, expressed MHC $\alpha$ and TnIc, and formed a fairly normal body axis with well-patterned head structures (Fig. 5A,B, panels e, $\mathrm{e}^{\prime}, \mathrm{f}^{\mathrm{f}} \mathrm{f}^{\prime}$ ). As expected, head structures were often inhibited in HexMo-injected DMZ explants (Fig. 5B, panels $\mathrm{g} \mathrm{g}^{\prime}$ ). As much as $100 \mathrm{ng}$ of ControlMo oligonucleotides could be injected with no reduction in cardiac marker expression (data not shown). Furthermore, cardiac differentiation was rescued by coinjection of mouse Hex mRNA, which cannot be blocked by the morpholino to Xenopus Hex (Fig. 5A,B, panels i,i'; from $31 \%$ to $52 \%$ and from $26 \%$ to $66 \%$ for incidences of MHC $\alpha$ and TNIC expression, respectively), confirming that the cardiac deficit observed following morpholino injection is specific to Hex.

HexMo also blocked ectopic, Dkk-1-dependent cardiogenesis in VMZ tissue (Fig. 5A,B, panels a-d'). Twenty nanograms of HexMo coinjected into two ventral blas-

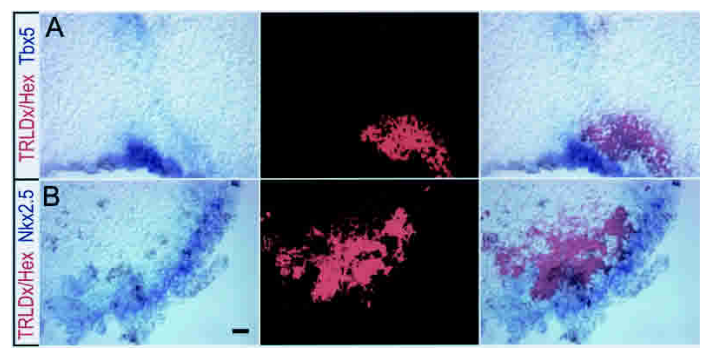

Figure 4. Non-cell-autonomous induction of $N k x 2.5$ and $T b x 5$ by Hex. $(A, B)$ Hex mRNA was injected with a fluorescent lineage tracer (TRLDx, 10,000 MW) with Hex into one ventral blastomere at the 16-32-cell stage as in Figure 1A. VMZ mesendoderm was explanted at stage 10.25-10.5, cultured to stage 23-25, and assayed for Tbx5 $(A)$ or $N k x 2.5(B)$. In situ hybridization is shown in the left panels, TRLDx fluorescence in the middle panels, and merged images on the right panels. Note gene induction at a distance from the region labeled with the lineage label. Bar in $B$ represents $20 \mu \mathrm{M}$. 
A
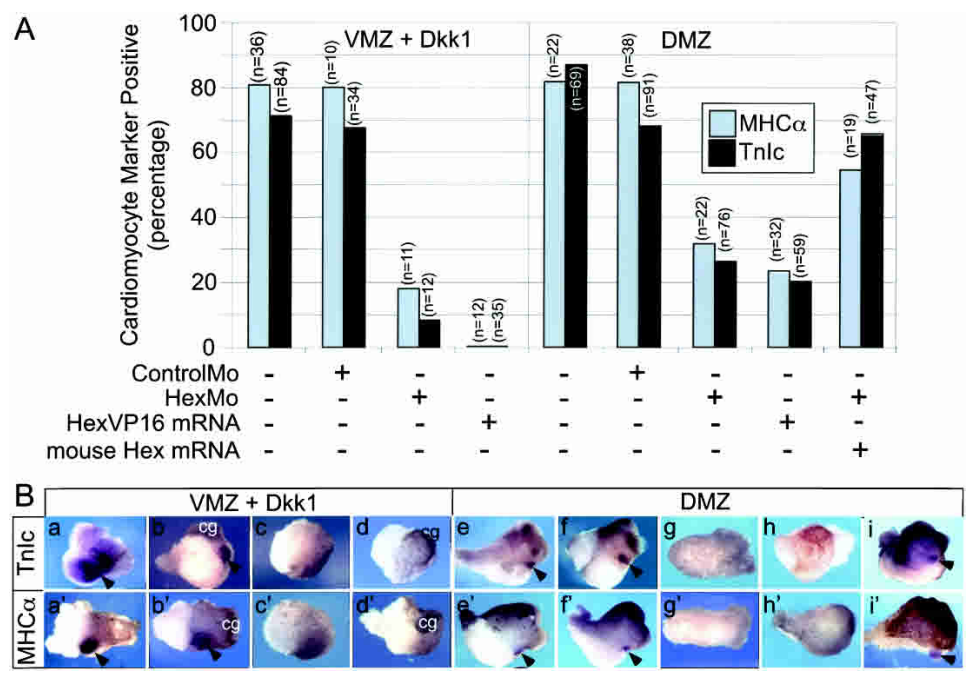

Figure 5. Hex is required for normal cardiogenesis and ectopic heart induction by Dkk-1. (A) Twenty nanograms of Hex antisense (HexMo), control (ControlMo) morpholino oligodeoxynucleotides, or an mRNA encoding a constitutively active Hex protein (HexVP16) was injected into two ventral or dorsal blastomeres of 4-cell-stage embryos (Materials and Methods). For ventral injections, Dkk-1 mRNA was included to induce ectopic cardiogenesis. VMZ or DMZ explants were prepared from ventrally or dorsally injected embryos, respectively, at the onset of gastrulation (stage 10.25-10.5) and maintained in culture to approximately stage 30 , when they were processed for in situ hybridization to TnIc or MHC $\alpha$. Note that antisense HexMo, but not ControlMo, attenuated cardiac marker expression in both DMZ and Dkk-1-injected VMZ, indicating that Hex is specifically required for normal and ectopic cardiogenesis. Hex has been characterized as both a transcriptional repressor and activator. HexVP16 mRNA also attenuated cardiogenesis and mimicked the Hex morpholino oligo-mediated depletion, indicating that the repressive function of Hex is required for cardiogenesis. Coinjection of an mRNA encoding a mouse Hex transcript, which is not recognized by the morpholino sequence, rescued both $M H C \alpha$ and TnIc expression, showing that the morpholino effect is specific to Hex. $(B)$ Examples of attenuation of both ectopic Dkk-1-induced cardiogenesis in VMZ explants (panels $a-d, a^{\prime}-d^{\prime}$ ) and normal cardiogenesis in DMZ explants (panels $e-i, e^{\prime}-i^{\prime}$ ). Explants stained for TnIc (panels $a-i$ ) or MHC $\alpha$ (panesl $a^{\prime}-i^{\prime}$ ). Uninjected DMZs (panels $e, e^{\prime}$ ), ControlMo-injected DMZs (panels $f, f^{\prime}$ ), and VMZs injected with DKK-1 either alone or with ControlMO (panels $\left.a, a^{\prime}, b, b^{\prime}\right)$ express cardiac markers in the heart region (arrowheads), whereas HexMo (panels $c, c^{\prime}, g, g^{\prime}$ ) or HexVP16 mRNA (panels $d, d^{\prime}, h, h^{\prime}$ ) injected explants frequently eliminated staining. Coinjection of mouse Hex mRNA rescued the cardiac deficit of Hex morpholino-injected DMZ explants (panels $i, i^{\prime}$ ), sometimes in the absence of recognizable head structures (panel $\left.i^{\prime}\right)$. cg denotes the highly pigmented cement glands that are present in DMZ explants and induced ectopically by Dkk-1 in VMZ tissues.

tomeres of a 4-cell-stage embryo along with Dkk-1 mRNA reduced the incidence of $M H C \alpha$ and TnIc expression in VMZ explants isolated at stage 10.25-10.5 and cultured to approximately stage 30 before analysis. Again, ControlMo (20 ng) had no significant effect. Based on this result and the fact that Dkk-1, Gsk3 $\beta$, and dnTCF3 each induces Hex mRNA, we conclude that Hex is a downstream effector of canonical Wnt signaling antagonists in heart induction.

\section{Transcriptional repressive activity of Hex is important} for heart induction

Hex has been characterized both as a transcriptional repressor and activator depending on cofactors (Brickman et al. 2000; Denson et al. 2000; Pellizzari et al. 2000; Nagai et al. 2001; Schaefer et al. 2001; Sekiguchi et al. 2001). To determine whether the activator or repressor function is responsible for heart induction, we used a construct in which the Hex protein is converted to a constitutive activator by fusion to a VP16 transactivation domain (Brickman et al. 2000). This construct was injected into two dorsoanterior blastomeres at the 4-cell stage. DMZ explants were isolated at stage 10-10.25 as above and placed in culture until approximately stage 30 . HexVP16 blocked heart marker expression as well as head development and axial patterning, whereas normal or control mRNA ( $\beta$-gal)-injected DMZ explants developed a fairly normal body axis, expressed heart markers, and exhibited well-patterned head structures (Fig. 5A,B, panels d, $\left.d^{\prime}, e, e^{\prime}\right)$. As for HexMo oligonucleotides, HexVP16 also blocked ectopic heart formation in experiments using Dkk-1-injected VMZ explants (Fig. 5A,B, panels h,h'). Since HexVP16 exhibited loss-of-function phenotype identical to that obtained with HexMo, we conclude that the repressive function of Hex is normally required for heart induction.

\section{Hex injection does not induce expression of late cardiac marker or beating heart tissue}

Finally, we asked if Hex might be the sole mediator of the heart-inducing activity of Dkk-1 or if the divergence of cytoplasmic signaling pathways at or downstream of the Frz receptor complex might induce other genes that act in parallel to Hex. As above, inducer mRNAs were injected independently into one ventral blastomere at the 4-cell stage, and VMZ explants were isolated at stage 10.25-10.5 and placed into culture until approximately stage 30 , when they were analyzed by quantitative RTPCR. Dkk-1, Gsk3 $\beta$, and dnTCF3 each induced late cardiac markers (MHC $\alpha$ and TnIc) (Schneider and Mercola 2001; data not shown); however, Hex never induced expression of late markers. Thus, we conclude that the divergent signaling, perhaps at the level of Dsh, GSK3 $\beta$, or $\beta$-catenin, activates parallel pathways that synergize with Hex to promote progression to later stages of heart differentiation. 


\section{Discussion}

Canonical Wnt/ $\beta$-catenin signaling antagonists such as Dkk-1, Crescent, and Wnt-11 have been thought to act directly on mesoderm in order to initiate cardiogenesis in Xenopus, chick, and possibly mouse embryos (Marvin et al. 2001; Schneider and Mercola 2001; Lickert et al. 2002; Pandur et al. 2002a), but cell autonomy has never been examined nor has there been any description of downstream effectors. Our experiments using cell-autonomous pathway antagonists support an indirect mode of action in which Wnt antagonists induce the homeodomain transcription factor Hex in endoderm, which, in turn, causes the production of a diffusible factor that acts back on the mesoderm to control induction of the heartforming region (Fig. 6). These results demonstrate a new role for the transcriptional repressing function of Hex as an essential effector of Wnt antagonists for heart induction. Our data also indicate that divergence of the Wnt signaling pathways, perhaps at the levels of Frz receptors, Dsh, Gsk3 $\beta$, or $\beta$-catenin, must activate genes besides Hex that control progression through the cardiogenic program.

\section{Hex and heart induction}

Hex (also known as Prh) was first identified as a divergent homeodomain-containing transcription factor expressed in hematopoietic progenitors, myeloid and liver cells (Crompton et al. 1992; Bedford et al. 1993; Hromas et al. 1993). It was later independently cloned from a germ-layer-specific cDNA library, isolated from gastrula-stage mouse embryos (Harrison et al. 1995) and subsequently shown to be one of the earliest markers of anteroposterior asymmetry in the developing mouse embryos, marking the AVE and subsequently the earliest emerging definitive endoderm (Thomas et al. 1998). Studies in which Xenopus Hex is inhibited, either by a dominant interfering construct (Brickman et al. 2000) or by antisense morpholinos (Smithers and Jones 2002), indicated that Hex acts cell autonomously in mesendoderm to delimit expression of Organizer-related genes such as goosecoid and chordin to more superficial tissue. These studies also suggested that Hex might regulate the production of diffusible molecules that convey patterning information to anterior mesoderm.

As shown by Arai et al. (1997), mouse AVE (and probably anterior-definitive endoderm as well) induces cardiogenesis when cocultured with primitive streak mesoderm. The visceral endoderm cell line END-2 similarly enhances the level of spontaneous cardiomyocyte differentiation in murine and human ES cells (Mummery et al. 2003), which, together with the spatiotemporal overlap in Hex expression with heart-inducing activity in Xenopus and chicks (Schneider and Mercola 1999; Yatskievych et al. 1999|, suggests an evolutionarily conserved cardiogenic function for Hex. Mice homozygous for a targeted disruption of $\mathrm{Hex}$ possess defects in forebrain patterning varying in severity from mild to severe (Martinez Barbera et al. 2000) and, additionally, have defects

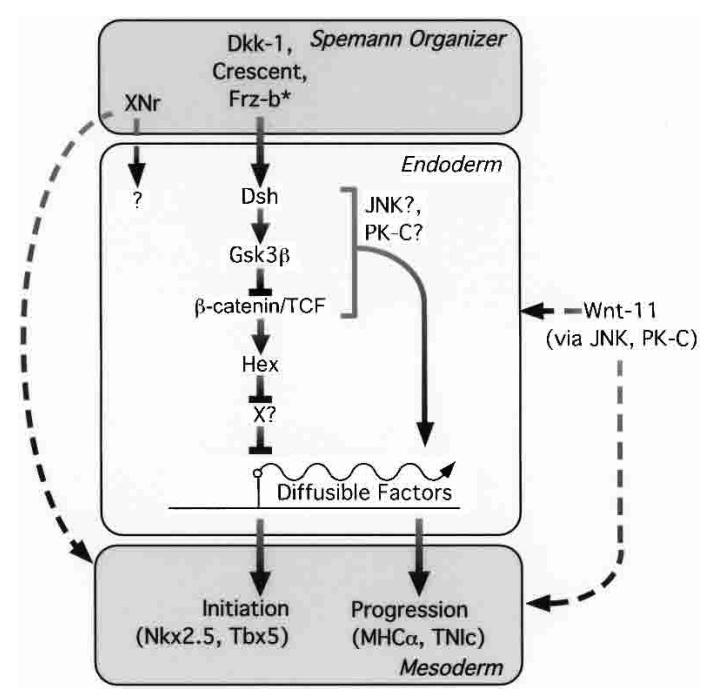

Figure 6. Molecular relay for heart induction. Inhibitors of canonical Wnt $/ \beta$-catenin signaling produced by the Organizer act on endoderm to induce Hex, which, in turn, controls the production of a diffusible factor that acts back on mesoderm to induce early cardiac genes (e.g., Nkx2.5 and Tbx5). Hex functions as a transcriptional repressor and might induce production of a diffusible activator by repression of a repressor (shown as $X$ ). Not depicted is the alternate scenario that Hex directly suppresses expression of a diffusible repressor of cardiogenesis. The finding that Hex induced early cardiac markers, whereas Gsk3 $\beta$ and dnTCF3 promoted progression to expression of myocardial markers (MHC $\alpha$ and TnIc), suggests that parallel pathways, perhaps involving activation of JNK and/or PK-C, regulate Hexindependent diffusible factors from the endoderm. Nodal related proteins $(\mathrm{XNr})$ and noncanonical Wnts might complement Hex-dependent heart induction, but it is not yet clear whether they act on endoderm or directly on cardiac mesoderm.

in liver, thyroid, and hematopoietic lineages (Keng et al. 2000; Martinez Barbera et al. 2000; Bogue et al. 2003). Although cardiac malformations were not described initially for Hex mutant mice (Martinez Barbera et al. 2000), mice harboring a more recently targeted allele (Hallaq et al. 2004) exhibit heart structural defects that might not have been noted in the original mice perhaps because of allelic differences or because the heart was not specifically examined at the relevant stages. The anomalies include defective vasculogenesis, hypoplasia of the right ventricle, overabundant endocardial cushions accompanied by ventricular septal defects, outflow tract abnormalities, and atrioventricular valve dysplasia and aberrant development of the compact myocardium. Because the targeted insertion affects Hex systemically, it is not yet possible to say whether the malformations are caused by an early deficit in heart induction or are secondary to defects elsewhere in the embryo. Heart formation, albeit anomalously, does proceed in these mice; thus, at the very least, the contrast with our finding that Hex is essential for Xenopus heart formation implicates partially redundant, compensatory, or parallel pathways in mice. 
Hex in the context of heart-inducing pathways

Our results position Hex as an essential effector of Wnt antagonists for the initiation of cardiogenesis, including expression of early genes (e.g., Nkx2.5 and Tbx5), but Hex did not induce myocardial markers (e.g., $M H C \alpha$ and $T n I c)$, suggesting that progression requires other signals. The Wnt signaling pathway diverges at several points, and the branches might control the production of multiple inducing factors from the endoderm (Fig. 6). Relevant to pathway divergence, Pandur et al. (2002a) have shown that Wnt 11, like Dkk-1, can induce cardiac tissue and further demonstrated that both factors repress canonical $\beta$-catenin signaling and activate a noncanonical JNK and PKC pathway in this setting. Pivotal proteins in mediating cross-talk between the canonical and other signaling pathways are the Frz receptors and Dishevelled (Dsh) (for review, see Pandur et al. 2002b; Wharton 2003), and a potential lack of JNK and PK-C signaling might explain our finding that dnTCF3 and Hex do not induce beating heart tubes as they lie downstream of Dsh. Divergence downstream of Dsh might also contribute, as Gsk3 $\beta$ and dnTCF3, but not Hex, induce late cardiac markers, perhaps reflecting contributions of Gsk3 $\beta$ substrates other than $\beta$-catenin or TCF/ LEF target genes other than Hex.

What proteins might complement the Hex-regulated heart-inducing signal? Different tissues provide heart-inducing stimuli across species, complicating an answer, yet, remarkably, many of the proteins involved are conserved. BMPs, for instance, are produced within heart field mesoderm in Xenopus but by underlying endoderm in chick (Schultheiss et al. 1997; Shi et al. 2000). Of potentially conserved signals, the TGF- $\beta$-family member Nodal is likely to cooperate with a Hex-dependent signal. Nodal proteins are broadly implicated in embryonic cardiogenesis (Logan and Mohun 1993; Sugi and Lough 1994; Yatskievych et al. 1997; Ladd et al. 1998; Griffin and Kimelman 2002), and the EGF-CFC protein Cripto, which potentiates Nodal signaling, promotes cardiogenic differentiation in mouse embryos and cultured mouse ES cells (Xu et al. 1998; Parisi et al. 2003). Whether Nodal/Cripto act on undifferentiated epiblast (or undifferentiated cells in the ES cultures), mesoderm, endoderm, or on multiple tissues is not entirely clear. Interestingly, induction of early cardiac markers (e.g., Nkx2.5) appears independent of Cripto in differentiating murine ES cells, while later markers of cardiomyocyte differentiation (e.g., $M H C \alpha$ ) appear dependent (Xu et al. 1998), suggesting that Nodal/Cripto signaling promotes cardiomyocyte differentiation from cardiac mesoderm and as such might complement the initiating activity of the Hex-dependent signal revealed by our study. On the other hand, Nodal might also act upstream of the Hexdependent signal. In the mouse, Nodal produced by the epiblast of the early-gastrula-stage embryo acts on the Hex-expressing distal visceral endoderm to promote proliferation and drive anteriorward movement, eventually up-regulating Nodal itself in this tissue (Arai et al. 1997; Varlet et al. 1997; Brennan et al. 2001; Norris et al. 2002;
Perea-Gomez et al. 2002; Yamamoto et al. 2004), and analogous signaling applies in chicks (Bertocchini and Stern 2002), where Nodal can up-regulate Hex (Yatskievych et al. 1999). Similarly, zebrafish genetic studies show that Nodal induces expression of the Sox-related transcription factor Casanova in early embryonic cells, thereby stimulating them to form endoderm (Aoki et al. 2002). These studies support a complex interaction between the Nodal and the Wnt antagonist/Hex pathways.

It seems, therefore, that vertebrates rely on a conserved set of signaling molecules and genes for heart induction, yet the strategies adopted for taking advantage of them differ. This might offer an explanation for the ability of hearts to develop in Hex mutant mice but not in Hex-deficient Xenopus. Cerberus expression spatially overlaps that of Hex and both induce early but not late cardiac gene expression in noncardiogenic mesendoderm and animal caps (herein and Bouwmeester et al. 1996). We found that Cerberus is not induced by Wnt antagonists, but others have shown it and mouse Cerberus-like to be up-regulated by Nodal (Ding et al. 1998; Brennan et al. 2001; Norris et al. 2002; Yamamoto et al. 2003). Although mouse Cerberus-like differs importantly from Xenopus Cerberus in its inability to bind Wnt (Belo et al. 2000), functional overlap between Hex and Cerberus or Cerberus-like might reinforce heart induction in Xenopus but constitute sufficient redundancy to cause only structural malformations in Hex mutant mice and perhaps contribute to absence of a heart phenotype in Cerberus-like mutants (Simpson et al. 1999; Belo et al. 2000; Shawlot et al. 2000).

\section{Materials and methods}

\section{Embryo and explant culture}

Embryos were fertilized in vitro, dejellied in $2 \%$ cysteine- $\mathrm{HCl}$ (pH 7.8), and maintained in 0.1× MMR (Peng 1991). Explant dissections were performed in $0.75 \times$ MMR using a fine tungsten needle. Embryos were staged according to Nieuwkoop and Faber (1967).

DMZ explants were dissected at stage 10.25-10.5, when the blastopore was clearly discernible. Explants to be examined for Hex or Cerberus expression were fixed in MEMFA for in situ hybridization or frozen for subsequent RNA isolation immediately following dissection. Explants to be examined for Nkx2.5 or Tbx 5 were grown in $0.75 \times$ MMR until age-matched siblings had reached stage 23-25 prior to processing. Explants to be examined for TnIc or MHC $\alpha$ were grown until age-matched siblings had reached approximately stage 30 .

\section{Morpholino and $m R N A$ injection}

Synthetic, capped mRNA for injection was transcribed from plasmids pSP6-nls- $\beta g a l$, pCS2-Dkk-1, pT7ts- $d n X T C F 3$ (a kind gift from Sergei Sokol, Mount Sinai School of Medicine, New

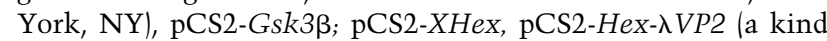
gift from Joshua Brickman, University of Edinburgh, Edinburgh, Scotland), and pCS2-XCer (a kind gift from Eddy deRobertis, University of California, Los Angeles, Los Angeles, CA) using SP6 and T7 mMessage kits (Ambion). All cDNAs used encode Xenopus proteins with the exception of the Hex- $\lambda$ VP2 con- 
struct, which contains the mouse coding sequence fused to two tandem VP16 activation domains (Brickman et al. 2000). The antisense Hex morpholino oligonucleotide was injected at $20 \mathrm{ng}$ and $100 \mathrm{ng}$ and is identical to the construct described in Smithers and Jones (2002); its sequence is 5'-GGTGCTGGTACTG CATGTCGATTCC-3'. The control morpholino was the standard control provided by Gene Tools. Morpholino rescue experiments were carried out by coinjection of mouse Hex mRNA (a kind gift from Joshua Brickman). This mouse transcript contains a divergent $5^{\prime}$ region upstream of the ATG that is not recognized by the Hex morpholino (Smithers and Jones 2002). Lineage studies were carried out by coinjection of mRNAs of interest with Texas Red lysinated dextran (10,000 MW, TRLDx; Molecular Probes), Alexa Fluor 546 dextran (10,000 MW, AD546; Molecular Probes), or nls- $\beta$-gal.

\section{Quantitative RT-PCR}

Ten explants were pooled from each round of injections, and RNA was produced using the Qiagen RNeasy Lipid Tissue Kit (QIAGEN). First-strand synthesis was carried out using a poly(dT) primer and Superscript II Reverse Transcriptase (Invitrogen). Real-time PCR was performed on a Roche Light Cycler using the Light Cycler FastStart DNA master SYBR Green I kit (Roche). Quantification was carried out by normalizing levels to the amount of total cDNA using the ubiquitously expressed $E F 1 \alpha$ as a standard. The EF1 $\alpha$ primer spans an intron, and PCR in the absence of RT thus confirmed that RNA samples were free from genomic DNA contamination. Q-RT-PCR was performed at least four times, and the two-sample $t$-test (assuming unequal variances) was performed to test the statistical significance of differences between samples.

The primer pairs were as follows: Cerberus, $\left(+\mid 5^{\prime}\right.$-CCTTGC CCTTCACTCAG-3' and (-) 5'-TGGCAGACAGTCCTTT-3'; EF1A, $(+)$ 5'-TACCCTCCTCTTGGTCGTT-3' and (-) 5'-GGTT TTCGCTGCTTTCTG-3'; Hex, (+) 5'-GTGGCTACTTACCG GAC-3' and (-) 5'-CCTTTCCGCTTGTGCA-3'; $M H C \alpha,(+) 5^{\prime}-$ CTGAGTCCCAGGTCAAC-3' and (-) 5'-GCTGAATTTAATG GTCACATTTTAT-3'; NKX2.5, (+) 5'-ATATGCGACGGTCA GA-3' and (-) 5'-GAGTGAAGCGACTAGGT-3'; TnIc, $(+)$ 5'AGAACACTGTCAGCCT- $3^{\prime}$ and $(-)$ 5'-AGATTGGCCCGTA GAT-3'.

\section{In situ hybridization and histology}

In situ hybridization was performed according to the protocol of Harland (1991). Digoxygenin-labeled probes were transcribed from the following linearized plasmid templates (restriction digest, polymerase): pBS-Xhex (BamHI, T7); pXMHC $\alpha$ (HindIII, T7); pGEM3Z-Nkx2.5 (XbaI, T7); pBS-Tbx5 (SacI, T7); and pXTnIc (Not1, T7). Following in situ hybridization, most explants were paraffin-embedded and sectioned for analysis.

\section{Acknowledgments}

We thank Clifford Bogue for communicating the cardiac phenotype of the targeted Hex mutant mice prior to publication and Joshua Brickman, Eddy de Robertis, and Sergei Sokol for providing cDNA clones. A.F. gratefully acknowledges the support of an NRSA fellowship (F32 HL69595), and M.M. gratefully acknowledges grant support from NIH NHLBI (R01 HL59502 and R01 HL67079).

\section{References}

Aoki, T.O., David, N.B., Minchiotti, G., Saint-Etienne, L., Dickmeis, T., Persico, G.M., Strahle, U., Mourrain, P., and Rosa,
F.M. 2002. Molecular integration of casanova in the Nodal signalling pathway controlling endoderm formation. Development 129: 275-286.

Arai, A., Yamamoto, K., and Toyama, J. 1997. Murine cardiac progenitor cells require visceral embryonic endoderm and primitive streak for terminal differentiation. Dev. Dyn. 210: 344-353.

Bedford, F.K., Ashworth, A., Enver, T., and Wiedemann, L.M. 1993. HEX: A novel homeobox gene expressed during haematopoiesis and conserved between mouse and human. Nucleic Acids Res. 21: 1245-1249.

Belo, J.A., Bachiller, D., Agius, E., Kemp, C., Borges, A.C., Marques, S., Piccolo, S., and De Robertis, E.M. 2000. Cerberus-like is a secreted BMP and nodal antagonist not essential for mouse development. Genesis 26: 265-270.

Bertocchini, F. and Stern, C.D. 2002. The hypoblast of the chick embryo positions the primitive streak by antagonizing nodal signaling. Dev. Cell 3: 735-744.

Bogue, C.W., Zhang, P.X., McGrath, J., Jacobs, H.C., and Fuleihan, R.L. 2003. Impaired B cell development and function in mice with a targeted disruption of the homeobox gene Hex. Proc. Natl. Acad. Sci. 100: 556-561.

Bouwmeester, T., Kim, S., Sasai, Y., Lu, B., and De Robertis, E.M. 1996. Cerberus is a head-inducing secreted factor expressed in the anterior endoderm of Spemann's organizer. Nature 382: 595-601.

Brennan, J., Lu, C.C., Norris, D.P., Rodriguez, T.A., Beddington, R.S., and Robertson, E.J. 2001. Nodal signalling in the epiblast patterns the early mouse embryo. Nature 411: 965969.

Brickman, J.M., Jones, C.M., Clements, M., Smith, J.C., and Beddington, R.S. 2000. Hex is a transcriptional repressor that contributes to anterior identity and suppresses Spemann organiser function. Development 127: 2303-2315.

Crompton, M.R., Bartlett, T.J., MacGregor, A.D., Manfioletti, G., Buratti, E., Giancotti, V., and Goodwin, G.H. 1992. Identification of a novel vertebrate homeobox gene expressed in haematopoietic cells. Nucleic Acids Res. 20: 5661-5667.

Denson, L.A., Karpen, S.J., Bogue, C.W., and Jacobs, H.C. 2000. Divergent homeobox gene hex regulates promoter of the $\mathrm{Na}^{+}$-dependent bile acid cotransporter. Am. J. Physiol. Gastrointest. Liver Physiol. 279: G347-G355.

Ding, J., Yang, L., Yan, Y.T., Chen, A., Desai, N., WynshawBoris, A., and Shen, M.M. 1998. Cripto is required for correct orientation of the anterior-posterior axis in the mouse embryo. Nature 395: 702-707.

Foley, A. and Mercola, M. 2004. Heart induction: Embryology to cardiomyocyte regeneration. Trends Cardiovasc. Med. 14: 121-125.

Fullilove, S.L. 1970. Heart induction: Distribution of active factors in newt endoderm. J. Exp. Zool. 175: 323-326.

Griffin, K.J. and Kimelman, D. 2002. One-Eyed Pinhead and Spadetail are essential for heart and somite formation. Nat. Cell Biol. 4: 821-825.

Hallaq, H., Pinter, E., Enciso, J., McGrath, J., Zeiss, C., Brueckner, M., Madri, J., Jacobs, H.C., Wilson, C.M., Vasavada, H., et al. 2004. A null mutation of Hhex results in abnormal cardiac development, defective vasculogenesis and elevated VEGFA levels. Development 131: 5197-5209.

Harland, R.M. 1991. In situ hybridization: An improved wholemount method for Xenopus embryos. Methods Cell Biol. 36: 685-695.

Harland, R. and Gerhart, J. 1997. Formation and function of Spemann's organizer. Annu. Rev. Cell Dev. Biol. 13: 611-667.

Harrison, S.M., Dunwoodie, S.L., Arkell, R.M., Lehrach, H., and Beddington, R.S. 1995. Isolation of novel tissue-specific 
genes from cDNA libraries representing the individual tissue constituents of the gastrulating mouse embryo. Development 121: 2479-2489.

Horb, M.E. and Thomsen, G.H. 1999. Tbx5 is essential for heart development. Development 126: 1739-1751.

Hromas, R., Radich, J., and Collins, S. 1993. PCR cloning of an orphan homeobox gene (PRH) preferentially expressed in myeloid and liver cells. Biochem. Biophys. Res. Commun. 195: 976-983.

Jacobson, A.G. 1960. Influences of ectoderm and endoderm on heart differentiation in the newt. Dev. Biol. 2: 138-154.

Jacobson, A.G. and Duncan, J.T. 1968. Heart induction in salamanders. J. Exp. Zool. 167: 79-103.

Kawano, Y. and Kypta, R. 2003. Secreted antagonists of the Wnt signalling pathway. J. Cell Sci. 116: 2627-2634.

Keng, V.W., Yagi, H., Ikawa, M., Nagano, T., Myint, Z., Yamada, K., Tanaka, T., Sato, A., Muramatsu, I., Okabe, M., et al. 2000. Homeobox gene Hex is essential for onset of mouse embryonic liver development and differentiation of the monocyte lineage. Biochem. Biophys. Res. Commun. 276: 1155-1161.

Ladd, A.N., Yatskievych, T.A., and Antin, P.B. 1998. Regulation of avian cardiac myogenesis by activin/TGF $\beta$ and bone morphogenetic proteins. Dev. Biol. 204: 407-419.

Larabell, C.A., Torres, M., Rowning, B.A., Yost, C., Miller, J.R., Wu, M., Kimelman, D., and Moon, R.T. 1997. Establishment of the dorso-ventral axis in Xenopus embryos is presaged by early asymmetries in $\beta$-catenin that are modulated by the Wnt signaling pathway. J. Cell Biol. 136: 1123-1136.

Lickert, H., Kutsch, S., Kanzler, B., Tamai, Y., Taketo, M.M., and Kemler, R. 2002. Formation of multiple hearts in mice following deletion of $\beta$-catenin in the embryonic endoderm. Dev. Cell 3: 171-181.

Logan, M. and Mohun, T. 1993. Induction of cardiac muscle differentiation in isolated animal pole explants of Xenopus laevis embryos. Development 118: 865-875.

Martinez Barbera, J.P., Clements, M., Thomas, P., Rodriguez, T., Meloy, D., Kioussis, D., and Beddington, R.S. 2000. The homeobox gene Hex is required in definitive endodermal tissues for normal forebrain, liver and thyroid formation. Development 127: 2433-2445.

Marvin, M.J., Di Rocco, G., Gardiner, A., Bush, S.M., and Lassar, A.B. 2001. Inhibition of Wnt activity induces heart formation from posterior mesoderm. Genes \& Dev. 15: 316-327.

Maye, P., Zheng, J., Li, L., and Wu, D. 2004. Multiple mechanisms for Wnt11-mediated repression of the canonical Wnt signaling pathway. J. Biol. Chem. 279: 24659-24665.

Molenaar, M., van de Wetering, M., Oosterwegel, M., PetersonMaduro, J., Godsave, S., Korinek, V., Roose, J., Destree, O., and Clevers, H. 1996. XTcf-3 transcription factor mediates $\beta$-catenin-induced axis formation in Xenopus embryos. Cell 86: 391-399.

Mummery, C., Ward-van Oostwaard, D., Doevendans, P., Spijker, R., van den Brink, S., Hassink, R., van der Heyden, M., Opthof, T., Pera, M., de la Riviere, A.B., et al. 2003. Differentiation of human embryonic stem cells to cardiomyocytes: Role of coculture with visceral endoderm-like cells. Circulation 107: 2733-2740.

Nagai, R., Suzuki, T., Aizawa, K., Miyamoto, S., Amaki, T., Kawai-Kowase, K., Sekiguchi, K.I., and Kurabayashi, M. 2001. Phenotypic modulation of vascular smooth muscle cells: Dissection of transcriptional regulatory mechanisms. Ann. NY Acad. Sci. 947: 56-66; discussion 66-67.

Nascone, N. and Mercola, M. 1995. An inductive role for the endoderm in Xenopus cardiogenesis. Development 121: 515-
523.

Newman, C.S., Chia, F., and Krieg, P.A. 1997. The XHex homeobox gene is expressed during development of the vascular endothelium: Overexpression leads to an increase in vascular endothelial cell number. Mech. Dev. 66: 83-93.

Nieuwkoop, P.D. and J. Faber. 1967. Normal table of Xenopus laevis (Daudin). A systematical and chronological survey of the development from the fertilized egg till the end of metamorphosis. North-Holland, Amsterdam.

Norris, D.P., Brennan, J., Bikoff, E.F., and Robertson, E.J. 2002. The Foxh1-dependent autoregulatory enhancer controls the level of Nodal signals in the mouse embryo. Development 129: 3455-3468.

Pandur, P., Lasche, M., Eisenberg, L.M., and Kuhl, M. 2002a. Wnt-11 activation of a non-canonical Wnt signalling pathway is required for cardiogenesis. Nature 418: 636-641.

Pandur, P., Maurus, D., and Kuhl, M. 2002b. Increasingly complex: New players enter the Wnt signaling network. Bioessays 24: 881-884.

Parisi, S., D'Andrea, D., Lago, C.T., Adamson, E.D., Persico, M.G., and Minchiotti, G. 2003. Nodal-dependent Cripto signaling promotes cardiomyogenesis and redirects the neural fate of embryonic stem cells. J. Cell Biol. 163: 303-314.

Pellizzari, L., D'Elia, A., Rustighi, A., Manfioletti, G., Tell, G., and Damante, G. 2000. Expression and function of the homeodomain-containing protein Hex in thyroid cells. Nucleic Acids Res. 28: 2503-2511.

Peng, H.B. 1991. Solutions and protocols. In Xenopus laevis: Practical uses in cell and molecular biology (eds. B.K. Kay and H.B. Peng), pp. 657-662. Academic Press, San Diego.

Perea-Gomez, A., Vella, F.D., Shawlot, W., Oulad-Abdelghani, M., Chazaud, C., Meno, C., Pfister, V., Chen, L., Robertson, E., Hamada, H., et al. 2002. Nodal antagonists in the anterior visceral endoderm prevent the formation of multiple primitive streaks. Dev. Cell 3: 745-756.

Sater, A.K. and Jacobson, A.G. 1990. The role of the dorsal lip in the induction of heart mesoderm in Xenopus laevis. Development 108: 461-470.

Schaefer, L.K., Wang, S., and Schaefer, T.S. 2001. Functional interaction of Jun and homeodomain proteins. J. Biol. Chem. 276: 43074-43082.

Schlange, T., Andree, B., Arnold, H.H., and Brand, T. 2000. BMP2 is required for early heart development during a distinct time period. Mech. Dev. 91: 259-270.

Schneider, V.A. and Mercola, M. 1999. Spatially distinct head and heart inducers within the Xenopus organizer region. Curr. Biol. 9: 800-809.

- 2001. Wnt antagonism initiates cardiogenesis in Xenopus laevis. Genes \& Dev. 15: 304-315.

Schultheiss, T.M., Burch, J.B., and Lassar, A.B. 1997. A role for bone morphogenetic proteins in the induction of cardiac myogenesis. Genes \& Dev. 11: 451-462.

Sekiguchi, K., Kurabayashi, M., Oyama, Y., Aihara, Y., Tanaka, T., Sakamoto, H., Hoshino, Y., Kanda, T., Yokoyama, T., Shimomura, Y., et al. 2001. Homeobox protein Hex induces SMemb/nonmuscle myosin heavy chain-B gene expression through the cAMP-responsive element. Circ. Res. 88: 52-58.

Shawlot, W., Min Deng, J., Wakamiya, M., and Behringer, R.R. 2000. The cerberus-related gene, Cerr1, is not essential for mouse head formation. Genesis 26: 253-258.

Shi, Y., Katsev, S., Cai, C., and Evans, S. 2000. BMP signaling is required for heart formation in vertebrates. Dev. Biol. 224: 226-237.

Simpson, E.H., Johnson, D.K., Hunsicker, P., Suffolk, R., Jordan, S.A., and Jackson, I.J. 1999. The mouse Cer1 (Cerberus re- 


\section{Foley and Mercola}

lated or homologue) gene is not required for anterior pattern formation. Dev. Biol. 213: 202-206.

Smithers, L.E. and Jones, C.M. 2002. Xhex-expressing endodermal tissues are essential for anterior patterning in Xenopus. Mech. Dev. 119: 191-200.

Sugi, Y. and Lough, J. 1994. Anterior endoderm is a specific effector of terminal cardiac myocyte differentiation of cells from the embryonic heart forming region. Dev. Dyn. 200: 155-162.

Thomas, P.Q., Brown, A., and Beddington, R.S. 1998. Hex: A homeobox gene revealing peri-implantation asymmetry in the mouse embryo and an early transient marker of endothelial cell precursors. Development 125: 85-94.

Tonissen, K.F., Drysdale, T.A., Lints, T.J., Harvey, R.P., and Krieg, P.A. 1994. XNkx-2.5, a Xenopus gene related to Nkx2.5 and tinman: Evidence for a conserved role in cardiac development. Dev. Biol. 162: 325-328.

Tzahor, E. and Lassar, A.B. 2001. Wnt signals from the neural tube block ectopic cardiogenesis. Genes \& Dev. 15: 255-260.

Varlet, I., Collignon, J., and Robertson, E.J. 1997. nodal expression in the primitive endoderm is required for specification of the anterior axis during mouse gastrulation. Development 124: 1033-1044.

Wharton Jr., K.A. 2003. Runnin' with the Dvl: Proteins that associate with Dsh/Dvl and their significance to Wnt signal transduction. Dev. Biol. 253: 1-17.

Xu, C., Liguori, G., Adamson, E.D., and Persico, M.G. 1998. Specific arrest of cardiogenesis in cultured embryonic stem cells lacking Cripto-1. Dev. Biol. 196: 237-247.

Yamamoto, S., Hikasa, H., Ono, H., and Taira, M. 2003. Molecular link in the sequential induction of the Spemann organizer: Direct activation of the cerberus gene by Xlim-1, Xotx2, Mix.1, and Siamois, immediately downstream from Nodal and Wnt signaling. Dev. Biol. 257: 190-204.

Yamamoto, M., Saijoh, Y., Perea-Gomez, A., Shawlot, W., Behringer, R.R., Ang, S.L., Hamada, H., and Meno, C. 2004. Nodal antagonists regulate formation of the anteroposterior axis of the mouse embryo. Nature 428: 387-392.

Yatskievych, T.A., Ladd, A.N., and Antin, P.B. 1997. Induction of cardiac myogenesis in avian pregastrula epiblast: The role of the hypoblast and activin. Development 124: 2561-2570.

Yatskievych, T.A., Pascoe, S., and Antin, P.B. 1999. Expression of the homebox gene Hex during early stages of chick embryo development. Mech. Dev. 80: 107-109. 


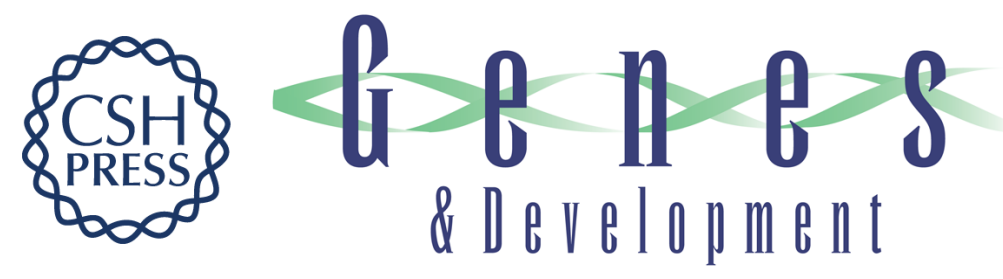

\section{Heart induction by Wnt antagonists depends on the homeodomain transcription factor Hex}

Ann C. Foley and Mark Mercola

Genes Dev. 2005, 19:

Access the most recent version at doi:10.1101/gad.1279405

References This article cites 61 articles, 18 of which can be accessed free at: http://genesdev.cshlp.org/content/19/3/387.full.html\#ref-list-1

License

Email Alerting

Receive free email alerts when new articles cite this article - sign up in the box at the top Service right corner of the article or click here.

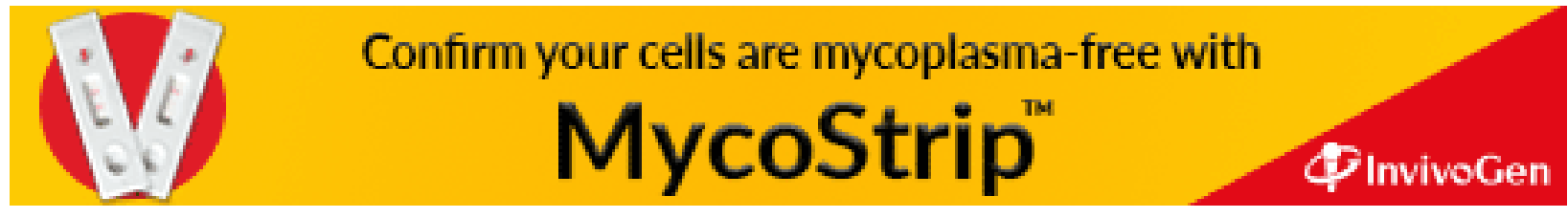

